\title{
Isolation of high $\gamma$-aminobutyric acid-producing lactic acid bacteria and fermentation in mulberry leaf powders
}

\author{
YANGSHENG ZHONG* ${ }^{*}$, SHAN WU*, FANGYAN CHEN, MENGXIU HE and JIANRONG LIN \\ College of Animal Science, South China Agricultural University, Guangzhou, Guangdong 510642, P.R. China
}

Received May 26, 2018; Accepted September 18, 2018

DOI: $10.3892 / \mathrm{etm} .2019 .7557$

\begin{abstract}
Amino butyric acid (GABA) has numerous roles in physiological processes, including neurotransmission, and induction of hypotensive, diuretic and tranquilizer effects. The present study aimed to produce GABA-enriched mulberry leaf powder by using a strain of high GABA-producing Lactobacillus pentosus SS6, which is isolated from fermented mulberry fruits. A total of 37 strains of lactic acid bacteria (LAB) were isolated from fermented mulberry fruits strains of high GABA-producing Lactobacillus pentosus were selected. The isolated LAB was analyzed using thin-layer chromatography. SS6 was used as a starter culture for the fermentation of mulberry leaf powder to produce GABA. The mulberry leaf powder was treated with $10 \%$ saccharose, $6 \%$ peptone, $1.6 \% \mathrm{~K}_{2} \mathrm{HPO}_{4}, 1 \% \mathrm{~L}$-sodium glutamate at $35^{\circ} \mathrm{C}$ for $36 \mathrm{~h}$ (each treatment was applied whilst the others were kept constant), in a mixture with a water content of $60 \%$, with the respective LAB strain that was fermented by incubation at $30^{\circ} \mathrm{C}$ for $6 \mathrm{~h}$. The results indicated that the SS6 strain produced significantly higher GABA contents in the fermentation broth compared to the other strains $(\mathrm{P}<0.05)$. Addition of $10 \%$ saccharose, $6 \%$ peptone, $1.6 \% \mathrm{~K}_{2} \mathrm{HPO}_{4}$ and $1 \%$ L-sodium glutamate significantly triggered the production of GABA compared with that in the groups void of those additives $(\mathrm{P}<0.05)$. Furthermore, the water content, treatment time, amount of LAB inoculated and the incubation temperature also significantly affected GABA production compared with untreated groups under the aforementioned conditions $(\mathrm{P}<0.05)$. In conclusion, $10 \%$ saccharose, $6 \%$ peptone, $1.6 \% \mathrm{~K}_{2} \mathrm{HPO}_{4}, 1 \% \mathrm{~L}$-sodium glutamate, and a $60 \%$ water content at $35^{\circ} \mathrm{C}$ significantly improved and enhanced GABA production. The present study provided a basis for the
\end{abstract}

Correspondence to: Dr Fangyan Chen, College of Animal Science, South China Agricultural University, 483 Wushan Road, Tianhe, Guangzhou, Guangdong 510642, P.R. China

E-mail: chenfangyan06@163.com

${ }^{*}$ Contributed equally

Key words: mulberry leaf, $\gamma$-aminobutyric acid, lactic acid bacteria, production production of GABA, which may be utilized by the pharmaceutical and food industry.

\section{Introduction}

$\gamma$-aminobutyric acid (GABA) is a non-protein amino acid that is synthesized by glutamic acid decarboxylase (GAD) (1,2). GABA has critical roles in the body, e.g., as an important inhibitory neurotransmitter in sympathetic nervous system, and supplementation of GABA has anti-diabetic and anti-hypertensive effects in humans (3). Furthermore, GABA suppresses anxiety and pain, controls the lipid levels and inhibits cancer cell proliferation and growth $(4,5)$. Following the clarification of the physiological roles of GABA, it was developed as a novel functional supplement and applied extensively in the pharmaceutical and food industry.

Natural GABA mainly occurs in the vegetables and fruit; however, only at low concentrations. Previous studies have reported that GABA is usually produced by numerous types of micro-organism, including yeasts, fungi and lactic acid bacteria (LAB) (5-7). Among these micro-organisms, LAB constitute the normal and resident flora in the gastrointestinal tract of humans and animals, which are also considered to be safe organisms by the food industry (8). LAB is utilized to improve the texture, and enhance the sensory profile and nutritional value of food or associated products (9). Of note, the GABA produced by LAB always exhibits higher biological activities compared with that in food products and its synthetic process is considered to be safe $(8,9)$. Therefore, the technology of GABA production by LAB has great potential for implementation in the health product and pharmaceutical industry, and is worthy of investigation.

In China, mulberry leaves have been used for $>5,000$ years (10), and are also being developed by the pharmaceutical industry. Of note, the extracts of mulberry leaves possess multiple biopharmaceutical activities, including anti-bacterial, anti-diabetic, anti-atherogenic, anticancer, anti-inflammatory and anti-oxidant effects, and have a therapeutic effect in cardiovascular and hypolipidemic diseases (11). GABA is an important active component of mulberry leaf extracts, from which it was isolated in a previous study (12). However, the enrichment of GABA in mulberry leaves is a critical problem that requires to be resolved for the production of GABA and its medical application. 
In order to enhance the yield of GABA isolated from mulberry leaves for its further exploration as a health product, the present study aimed to obtain GABA-enriched mulberry leaf powders by using a strain of high GABA-yielding Lactobacillus pentosus SS6 isolated from fermented mulberry fruits.

\section{Materials and methods}

Regents and materials. GABA was purchased from Sigma-Aldrich (Merck KGaA, Darmstadt, Germany). The nutrient agars including de Man Rogosa and Sharpe (MRS), tryptone extract glucose (TYG) and lactobacillus isolation medium) were purchased from Guangdong Huankai Microbial Sci. Tech. Co. Ltd. (Guangzhou, China). Peptone cat. No. LP0017T), tryptone (cat. no. CM0595B), yeast extract (cat. no. LP0021B) and beef extract (cat. no. CM0015B) were purchased from Beijing BioDee Biotech. Co. Ltd. (Beijing, China). Massif blue G-250, glutamate, sodium glutamate, $\mathrm{K}_{2} \mathrm{HPO}_{4}$, mercaptoethanol, absolute ethyl alcohol, formaldehyde, methanol, acetone, hydrochloric were purchased from Sigma-Aldrich (Merck KGaA). The mulberry leaves were provided by Serigen Group (Guangzhou, China). The mulberry leaves were turned into mulberry leaf powder by New Toldhow Pharmaceutical Co. Ltd. (Jiangxi, China). The fresh mulberries were purchased from Guangdong BSYCY Co. Ltd. (Guangzhou, China).

Preparation of culture medium. The isolation of LAB and preparation of culture medium were performed according to the protocol of a previous study (13), following sterilization via autoclave for $25 \mathrm{~min}$ and adjustment of the $\mathrm{pH}$ to 6.5 (13). The tryptone yeast extract glucose (TYG) fermentation medium was prepared according to a previous study (14), with the modifications of addition of $2 \% \mathrm{~L}-\mathrm{Glu}(1 \mathrm{mg} / \mathrm{ml})$, adjustment of the $\mathrm{pH}$ to 6.5 and sterilization for $25 \mathrm{~min}$. The LAB medium was composed of skimmed milk (12 g) and $\mathrm{CaCO}_{3}(0.2 \mathrm{~g})$; sterilization was performed for $25 \mathrm{~min}$ and the $\mathrm{pH}$ was adjusted to 6.5.

Isolation, culture and screening of LAB. Isolation of LAB from the fermented mulberries ( $5 \mathrm{~g}$ ) was performed as previously described (15). The LAB was cultured according to a previous study (16). In brief, the isolated LAB was cultured in TYG culture medium at $37^{\circ} \mathrm{C}$ for $48 \mathrm{~h}$. The LAB screening was performed according to the study by $\mathrm{Wu}$ and Shah (17) with the following modifications: Supernatant fermentation liquid $(1 \mu \mathrm{l})$ was added to the thin-layer chromatography silica gel plate (cat. No. SG-004; Qingdao Kangyexin Medical Silica Gel Desiccant Co., Ltd., Qingdao, China). n-butyl alcohol: Glacial acetic acid: Water $(4: 1: 3)$ was considered as the developing agent. The $0.4 \%$ ninhydrin (Sigma-Aldrich; Merck $\mathrm{KGaA}$ ) was assigned as the chromogenic agent. The isolated LAB was cultured in the TYG culture medium and screened by using a ninhydrin colorimetry method [0.4\% ninhydrin; Sigma-Aldrich (Merck KGaA)] (16). In brief, TYG with added GABA (1 mg/ml) was used as a positive control and TYG without any reagents was used as a negative control.

Carbon source and nitrogen source treatment. The isolated LAB were added to the mulberry leaf powder $(100 \mathrm{~g})$ culture (water content, 60\%) at a proportion of 5\%. Glucose, saccharose or xylose was used as the carbon source, which was individually added at concentrations of $0,5,10$ or $15 \%$. In another experiment, peptone was used as the nitrogen source, which was added at concentrations of $0,2,4,6$ or $8 \%$. Subsequent to the abovementioned additions, the mulberry leaf powder mixtures were fermented via shaking for $36 \mathrm{~h}$ at $35^{\circ} \mathrm{C}$ using a $500 \mathrm{ml}$ flask (Mode, 4100-0500; Pierce; Thermo Fisher Scientific, Inc., Waltham, MA, USA).

$\mathrm{K}_{2} \mathrm{HPO}_{4}$ treatment. The isolated $\mathrm{LAB}$ was added to the mulberry leaf powder culture (with a water content of $60 \%$ ) at a final LAB concentration of $5 \%$. For the $\mathrm{K}_{2} \mathrm{HPO}_{4}$ treatment, $\mathrm{K}_{2} \mathrm{HPO}_{4}$ was added at concentrations of $0,0.4,0.8,1.2,1.6,3$, 4 or $6 \%$, followed by fermentation for $36 \mathrm{~h}$ at $35^{\circ} \mathrm{C}$.

L-sodium glutamate treatment. To the LAB/mulberry leaf powder culture as specified above, L-sodium glutamate was added at a concentration of $0,0.5,1,1.5$ or $3 \%$, followed by fermentation as stated above.

Effects of water content, fermentation time, LAB inoculated amount and temperature on the GABA content. Various experimental conditions were examined in order to determine those leading to the highest yield of GABA. The water content was set at $30,40,50,60$ or $70 \%$. The time of fermentation was $0,12,24,36$ or $48 \mathrm{~h}$. The amount of inoculated LAB was $0,1,3,5,7$ or $9 \%$. The temperature was set at $25,30,35$ or $43^{\circ} \mathrm{C}$. In the preliminary experiments, the aforementioned varied LAB content, water content and temperature were utilized. However, the pre-experiment results revealed that the time point of $36 \mathrm{~h}$ and an LAB content of 35 and $5 \%$ were the optimal treatments for the current study. Therefore, the mulberry leaf powders were fermented for $36 \mathrm{~h}$ at $35^{\circ} \mathrm{C}$ for each type of treatment.

Measurement of GABA content. The GABA content was evaluated by using the Merck Purospher STAR RP-18e high-performance liquid chromatography system (Merck $\mathrm{KGaA}$ ) equipped with a reverse-phase column with $5 \mu \mathrm{m}$ external diameter, $4.6 \mathrm{~mm}$ internal diameter and $250 \mathrm{~mm}$ length (Mode, Luna C18; Phenomenex, Inc., Torrance, CA, USA). The processes for the GABA content measurement were according to those of a previous study (18). The GABA content was determined by comparing the peak area with that of the associated GABA standard. Furthermore, mycelium biomass was assessed using the colony-counting method (colony formation unit counting) as previously described (19).

Statistical analysis. All of the data were analyzed by using SPSS software 22.0 (IBM Corp., Armonk, NY, USA). The quantitative data were expressed as the mean \pm standard deviation. Student's t-test was used for analysis of differences between two groups. Analysis of variance and Tukey's post-hoc test were used for comparing measurement data between multiple groups. All of the data were obtained from at least six independent measurements or experiments. $\mathrm{P}<0.05$ was considered to indicate a statistically significant difference. 
Table I. Physiocochemical indexes of fermentation product.

\begin{tabular}{|c|c|c|}
\hline Parameter & $\begin{array}{l}\text { Mulberry leaf powder mixture } \\
\text { prior to fermentation }\end{array}$ & $\begin{array}{l}\text { Fermented and enriched } \\
\text { mulberry leaf powder }\end{array}$ \\
\hline $\mathrm{GABA}(\mathrm{mg} / \mathrm{g})$ & $10.7 \pm 0.04$ & $54.96 \pm 0.02^{\mathrm{a}}$ \\
\hline $\mathrm{pH}$ & $6.256 \pm 0.01$ & $5.9 \pm 0.06$ \\
\hline Acidity (\%) & $1.35 \pm 0.07$ & $4.77 \pm 0.11^{\mathrm{b}}$ \\
\hline Reduced sugar $(\mathrm{mg} / \mathrm{g})$ & $0.66 \pm 0.01$ & $0.23 \pm 0.01^{\mathrm{b}}$ \\
\hline Total sugar $(\mathrm{mg} / \mathrm{g})$ & $54.95 \pm 0.06$ & $32.86 \pm 0.04^{\mathrm{a}}$ \\
\hline Flavone (mg/g) & $14.93 \pm 0.01$ & $15.85 \pm 0.01$ \\
\hline Protein $(\mathrm{mg} / \mathrm{g})$ & $6.76 \pm 0.03$ & $6.72 \pm 0.03$ \\
\hline Coarse fiber $(\mathrm{mg} / \mathrm{g})$ & $219.1 \pm 0.31$ & $205.4 \pm 0.17$ \\
\hline Mycelium biomass $[\log (\mathrm{cfu} / \mathrm{g})]$ & - & $3.95 \times 10^{9}$ \\
\hline
\end{tabular}

${ }^{\mathrm{a}} \mathrm{P}<0.01$; ${ }^{\mathrm{b}} \mathrm{P}<0.05$ vs. mulberry leaf powder mixture prior to fermentation. GABA, $\gamma$-aminobutyric acid; cfu, colony-forming units.

\section{Results}

Screening of the GABA-producing $L A B$. In the present study, 6 strains among a total of 37 LAB strains were selected for producing GABA using thin layer chromatography, as they exhibited GABA production at the same gel position. The preliminary results indicated that the supernatants of the fermentation culture of the SC6 and SS6 strains exhibited higher levels of GABA than that of the others (Fig. 1A). However, compared with the SC6 strain, the SS6 stain exhibited higher levels of GABA (Fig. 1). Therefore, the SS6 strain was employed to evaluate the production of GABA in the subsequent experiments.

The SS6 strain produces the highest GABA content in the fermentation broth. In order to evaluate the production of GABA induced by the LAB strains, the LB2, LB6, SC5, SC6, SC9 and SS6 Lactobacillus pentosus strains were cultured. The results indicated that the yield of GABA in the culture with the SS6 strain was significantly higher than that in the cultures of the other strains $(\mathrm{P}<0.05$; Fig. 1B). The SS6 LAB was also uniquely prepared using MRS and isolation culture (Fig. 1C), and was employed in the subsequent experiments.

Fermentation and enrichment of mulberry leaf powder by $L A B$ enhances the yield of GABA. As presented in Table I, after the fermentation of mulberry leaf powder by the SS6 strain, the culture contained significantly higher levels of GABA compared with those in the unfermented mulberry leaf powder mixture $(\mathrm{P}<0.05)$. Furthermore, the $\mathrm{pH}$ value of the mulberry leaf powder culture after fermentation was lower compared with that of the unfermented mulberry leaf powder mixture. Furthermore, the amounts of reduced sugar and total sugar in the fermented mulberry leaf powder were significantly lower compared with those in the unfermented mulberry leaf powder $(\mathrm{P}<0.05)$.

Addition of $10 \%$ saccharose as the optimal carbon source causes the greatest enhancement of GABA production. The production of GABA and mycelium biomass was evaluated in the cultures treated with glucose, saccharose or xylose at different concentrations as carbon sources. The results indicated that addition of saccharose, particularly at 5 and $10 \%$, resulted in a higher production of GABA compared with that achieved with glucose and xylose treatment $(\mathrm{P}<0.05$; Fig. $2 \mathrm{~A})$. The mycelium biomass in the saccharose group (particularly the $10 \%$ group) was also significantly higher compared with that in the glucose and xylose groups $(\mathrm{P}<0.05$; Fig. $2 \mathrm{~B})$. Therefore, saccharose at $10 \%$ was determined to be the optimal carbon source.

Addition of $6 \%$ peptone as a nitrogen source enhances $G A B A$ production. The results indicated that the GABA production in the $6 \%$ peptone group was significantly higher compared with that in the groups treated with other concentrations of peptone $(\mathrm{P}<0.05$; Fig. 3A). However, the mycelium biomass was highest in the $4 \%$ peptone-treated group, which suggests that the GABA production is not consistent with the mycelium biomass.

Addition of $1.6 \% \mathrm{~K}_{2} \mathrm{HPO}_{4}$ enhances the production of $G A B A$. The results indicated that the GABA production in the $1.6 \% \mathrm{~K}_{2} \mathrm{HPO}_{4}$ treatment group was significantly higher compared with that in the other $\mathrm{K}_{2} \mathrm{HPO}_{4}$ treatment groups $(\mathrm{P}<0.05$; Fig. 3B). However, the mycelium biomass in the $0.8 \% \mathrm{~K}_{2} \mathrm{HPO}_{4}$ treatment group was higher than that in the other groups $(\mathrm{P}<0.05$; Fig. $3 \mathrm{~B})$.

Addition of $1 \%$ L-sodium glutamate enhances the production of $G A B A$. L-sodium glutamate was added to the mulberry leaf powder culture in the experiments. The results indicated that L-sodium glutamate at the concentration of $1 \%$ had the greatest capacity to enhance the production of GABA compared with the other concentrations $(\mathrm{P}<0.05$; Fig. $3 \mathrm{C})$. However, the highest mycelium biomass was obtained with L-sodium glutamate at $0.5 \%$ (Fig. 3C).

Water content and treatment time affect GABA production. The results indicated that a water content of $60 \%$ led to the highest GABA production and mycelium biomass compared to the other concentrations $(\mathrm{P}<0.05$; Fig. $4 \mathrm{~A})$. Furthermore, the GABA production and mycelium biomass were highest 
A
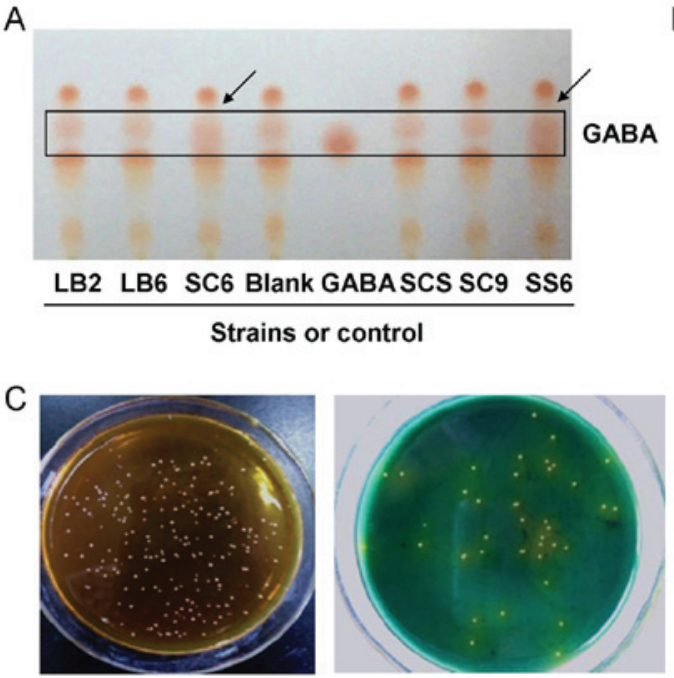

MRS culture

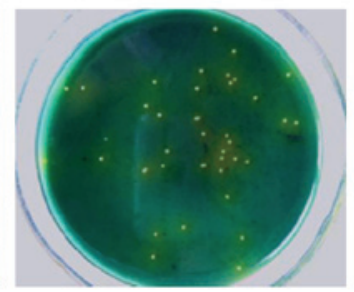

Isolated culture

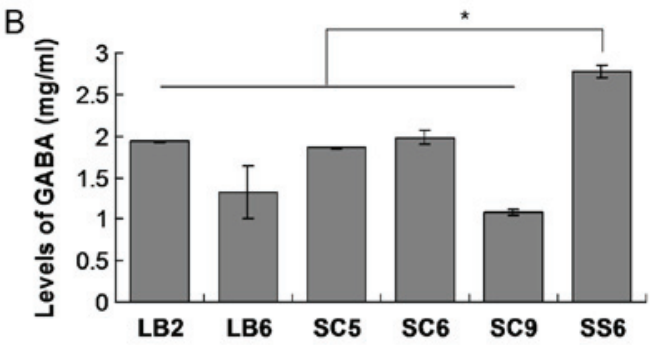

Figure 1. Isolation and identification of lactic acid bacteria. (A) Thin-layer chromatogram of different bacterial strains. Strain SS6 produced the greatest amount of GABA (arrows). (B) The levels of GABA produced by bacterial strains. (C) Morphology of strain SS6 in MRS culture and isolated culture. *P $<0.05$, SS6 group vs. LB2, LB6, SC5, SC6 or SC9 group. GABA, $\gamma$-aminobutyric acid; MRS, de Man, Rogosa and Sharpe agar.

A

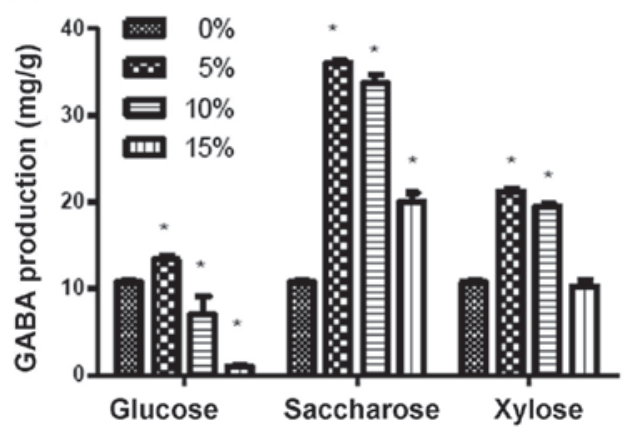

B

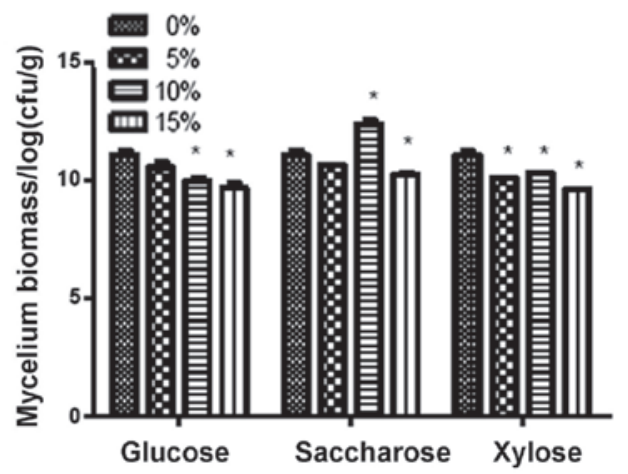

Figure 2. Effects of different carbon sources on the levels of GABA and bacterial biomass concentration. (A) Levels of GABA production in groups incubated with different percentages of various sugars. (B) Bacterial biomass in the different groups. ${ }^{*}<0.05$ vs. the respective $0 \%$ sugar group. GABA, $\gamma$-aminobutyric acid; cfu, colony-forming units.

if the incubation time the mulberry leaf powder culture was 36 h (Fig. 4B).

The amount of $L A B$ and the culture temperature affect $G A B A$ production. According to the weight/volume ratio, $7 \%$ LAB amounts used for inoculation achieved a significantly higher GABA production compared with the other amounts assessed $(\mathrm{P}<0.05$; Fig. 4C). Furthermore, among all incubation temperatures, the highest levels of GABA production and mycelium biomass were achieved at $35^{\circ} \mathrm{C}$ (Fig. 4D).

\section{Discussion}

LAB have been reported to produce a series of metabolites, including bacteriocins (20), vitamins (21), conjugated linoleic acid (22) and exopolysaccharides (23), all of which are always considered as the basis of probiotic function. A potential metabolite produced by the lactobacilli in the intestinal tract is GABA, which is linked to the desirable effects on the host (24). Previous studies have indicated that GABA has roles in maintaining homoeostasis and in the brain-gut-microbiome axis $(25,26)$. The present study aimed to isolate a high GABA-yielding LAB strain from mulberries and explore the optimal conditions for obtaining GABA from a fermentation/ enrichment culture of mulberry leaf powder with LAB and various additives.

In the present study, the isolated SS6 strain achieved the highest yield of GABA, and was therefore used in the subsequent experiments. In order to determine the production of GABA induced by the different LAB strains, the LB2, LB6, SC5, SC6, SC9 and SS6 strains were used in fermentation/enrichment culture with mulberry leaf powder. Comparison of the different strains indicated that use of the SS6 strain achieved the highest yield of GABA, which was therefore considered to be the best candidate for the improvement for the GABA content and production. The SS6 strain was used in the subsequent experiments for exploring the ideal conditions for the mulberry leaf powder fermentation culture.

Apart from the production of GABA through chemical synthesis in the laboratory, it may also be produced by 

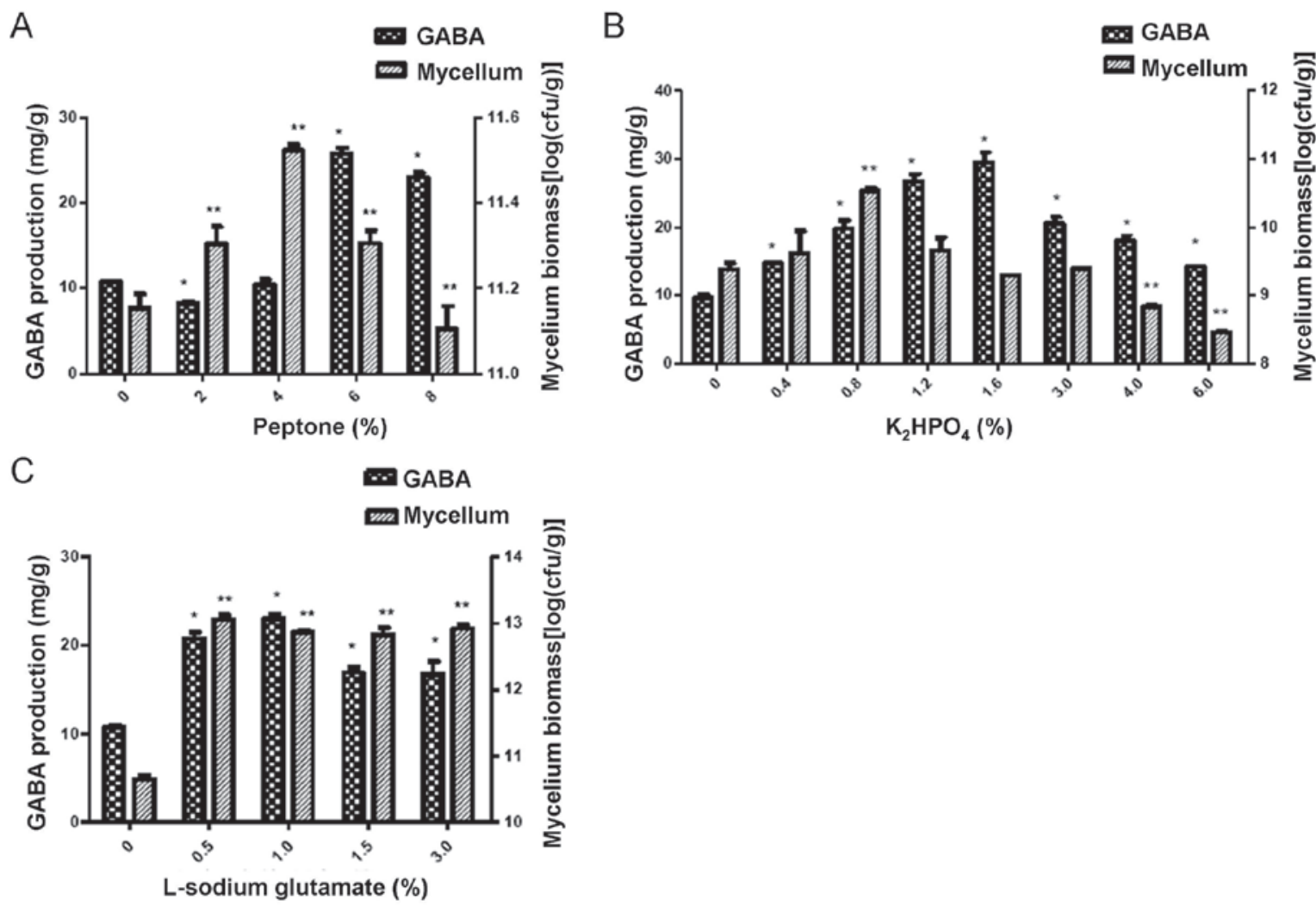

Figure 3. Effects of peptone (nitrogen source), $\mathrm{K}_{2} \mathrm{HPO}_{4}$ and L-sodium glutamate in the solid-state fermentation on GABA production and bacterial biomass (A) Peptone treatment, (B) $\mathrm{K}_{2} \mathrm{HPO}_{4}$ treatment and (C) L-sodium glutamate treatment. ${ }^{*} \mathrm{P}<0.05$, ${ }^{* *} \mathrm{P}<0.01$ vs. $0 \%$ peptone, $0 \% \mathrm{~K}_{2} \mathrm{HPO}_{4}$ or $0 \% \mathrm{~L}$-sodium glutamate. GABA, $\gamma$-aminobutyric acid; cfu, colony-forming units.

A

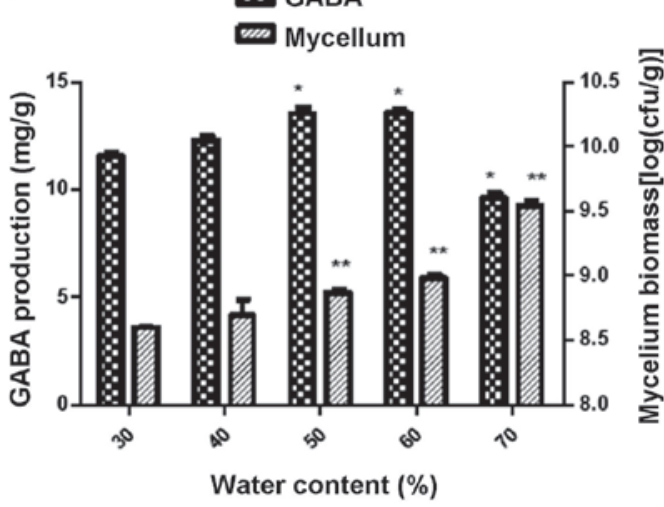

C

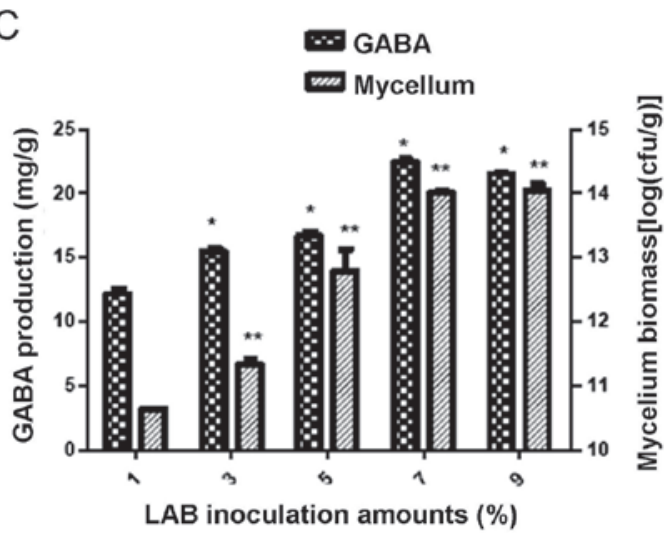

B
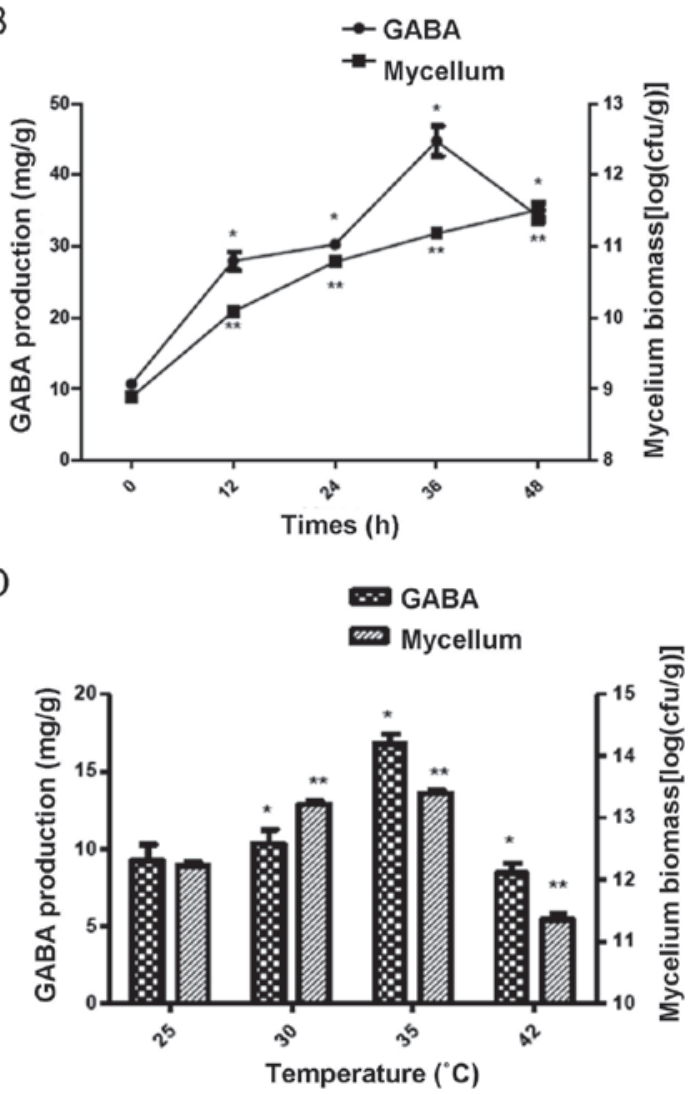

Figure 4. Effects of water content, time, inoculation concentration and temperature on the solid-state fermentation. (A) Water content, (B) treatment time, (C) inoculation concentration and (D) incubation temperature. ${ }^{*} \mathrm{P}<0.05,{ }^{* *} \mathrm{P}<0.01$ vs. $30 \%$ water content, $0 \mathrm{~h}, 1 \%$ inoculation amounts or a temperature of $25^{\circ} \mathrm{C}$. GABA, $\gamma$-aminobutyric acid; cfu, colony-forming units. 
fermentation of biomaterials containing GABA, e.g., mulberry leaves, in the presence of LAB. Of note, the production of GABA is affected by different culture and fermentation conditions, which require optimization. In the present study, the effects of the addition of a carbon source, a nitrogen source, $\mathrm{K}_{2} \mathrm{HPO}_{4}$ and L-sodium glutamate, as well as variation of the water content, treatment time, amount of inoculated LAB and the incubation temperature, on the production of GABA were investigated, all of which were previously indicated to affect the yield of GABA $(27,28)$. The results of the present study demonstrated that $10 \%$ saccharose, $6 \%$ peptone, $1.6 \% \mathrm{~K}_{2} \mathrm{HPO}_{4}$ and $1 \%$ L-sodium glutamate achieved the highest GABA production and/or mycelium biomass compared with those at the other concentrations. These results were comparable to those of previous studies, which reported that the production of GABA is affected by LAB and also by other micro-organisms (yeast) $(29,30)$.

An important characteristic of probiotic bacteria is that they have beneficial effects in the host. In the present study, LAB produced GABA, which has potential health benefits and a promising application in the pharmaceutical and food industry.

Although the present study provided numerous useful results, it also had a few limitations. First, the present study has not addressed whether the production of GABA by the original strains is consistent with that by the strains after undergoing multiple passages. Second, the current study only changed one variable at a time, with all others remaining constant. The combination of constant variables however, does not guarantee that the highest yield is produced. For example, the addition of $\mathrm{K}_{2} \mathrm{HPO}_{4}$ may alter the $\mathrm{pH}$, which may then effect other components. In further study, the different factors that may affect GABA production will be considered.

In conclusion, the present study isolated the SS6 LAB strain from fermented mulberries. The strain was cultured with mulberry leaf powder for producing GABA. In summary, $10 \%$ saccharose, $6 \%$ peptone, $1.6 \% \mathrm{~K}_{2} \mathrm{HPO}_{4}, 1 \% \mathrm{~L}$-sodium glutamate, as well as the appropriate water content and temperature significantly improved and enhanced GABA production and the mycelium biomass. In future study, combined reactions (involving all the aforementioned factors) in which all conditions are applied at the same time will be assessed. The present study provided a basis for the production of GABA by the pharmaceutical and food industry.

\section{Acknowledgements}

Not applicable.

\section{Funding}

The present study was supported by the Scientific Research Project of Public Welfare Industry (Agriculture) of the Ministry of Agriculture (grant no. 201403064) and the Guangdong Science and Technology Project (grant no. 2017A020208044).

\section{Availability of data and materials}

All data generated or analyzed during this study are included in this published article.

\section{Authors' contributions}

YZ, FC and MH performed the experiments. SW performed the experiments and wrote the manuscript. FC designed the study and wrote the manuscript. JL assisted in the study design. All authors read and approved the final manuscript.

\section{Ethical approval and consent to participate}

Not applicable.

\section{Patient consent for publication}

Not applicable.

\section{Competing interests}

Authors declare no competing financial or commercial interests regarding this study.

\section{References}

1. Yu T, Jiang Z, Liu L and Fan Z: Decrease of gamma-aminobutyric acid and zinc ions in the islet periportal circulation stimulates glucagon secretion during hypoglycemia. Exp Ther Med 15: 2507-2511, 2018.

2. Kondoh T, Mallick HN and Torii K: Activation of the gut-brain axis by dietary glutamate and physiologic significance in energy homeostasis. Am J Clin Nutr 90 (Suppl): 832S-837S, 2009.

3. Wong CG, Bottiglieri $T$ and Snead OC III: GABA, gamma-hydroxybutyric acid, and neurological disease. Ann Neurol 54 (Suppl 6): S3-S12, 2003.

4. Miura D, Ito Y, Mizukuchi A, Kise M, Aoto H and Yagasaki K: Hypocholesterolemic action of pre-germinated brown rice in hepatoma-bearing rats. Life Sci 79: 259-264, 2006.

5. Park KB and Oh SH: Production of yogurt with enhanced levels of gamma-aminobutyric acid and valuable nutrients using lactic acid bacteria and germinated soybean extract. Bioresour Technol 98: 1675-1679, 2007.

6. Kim JY, Lee MY, Ji GE, Lee YS and Hwang KT: Production of gamma-aminobutyric acid in black raspberry juice during fermentation by Lactobacillus brevis GABA100. Int J Food Microbiol 130: 12-16, 2009.

7. Chuang CY, Shi YC, You HP, Lo YH and Pan TM: Antidepressant effect of GABA-rich monascus-fermented product on forced swimming rat model. J Agric Food Chem 59: 3027-3034, 2011.

8. Barthelmebs L, Calas-Blanchard C, Istamboulie G, Marty JL and Noguer T: Biosensors as analytical tools in food fermentation industry. Adv Exp Med Biol 698: 293-307, 2010.

9. Lucke FK: Utilization of microbes to process and preserve meat. Meat Sci 56: 105-115, 2000

10. Wang D, Dong Z, Zhang Y, Guo K, Guo P, Zhao P and Xia Q: Proteomics provides insight into the interaction between mulberry and silkworm. J Proteome Res 16: 2472-2480, 2017.

11. Gryn-Rynko A, Bazylak G and Olszewska-Slonina D: New potential phytotherapeutics obtained from white mulberry (Morus alba L.) leaves. Biomed Pharmacother 84: 628-636, 2016.

12. Chen H, He X, Liu Y, Li J, He Q, Zhang C, Wei B, Zhang Y and Wang J: Extraction, purification and anti-fatigue activity of gamma-aminobutyric acid from mulberry (Morus alba L.) leaves. Sci Rep 6: 18933, 2016.

13. Süle J, Kõrösi T, Hucker A and Varga L: Evaluation of culture media for selective enumeration of bifidobacteria and lactic acid bacteria. Braz J Microbiol 45: 1023-1030, 2014.

14. Zhao Z, Wei L, Li CY, Wang Z, Hu YW, Liu CC and Ma F: Study on optimization of proportion between fermented liquid and traditional cultural medium of bioflocculant production and its flocculant performance considering the aerobic fermentation of rice straw as substrate. Pak J Pharm Sci 27 (6 Suppl): S2119-S2123, 2014. 
15. Lee HJ, Lee H, Choi YI and Lee JJ: Effect of lactic acid bacteria-fermented mulberry leaf extract on the improvement of intestinal function in rats. Korean J Food Sci Anim Resour 37: 561-570, 2017.

16. Tongpim S, Meidong R, Poudel P, Yoshino S, Okugawa Y, Tashiro Y, Taniguchi M and Sakai K: Isolation of thermophilic L-lactic acid producing bacteria showing homo-fermentative manner under high aeration condition. J Biosci Bioeng 117: 318-324, 2014.

17. Wu Q and Shah NP: Gas release-based prescreening combined with reversed-phase HPLC quantitation for efficient selection of high-gamma-aminobutyric acid (GABA)-producing lactic acid bacteria. J Dairy Sci 98: 790-797, 2015.

18. Tajabadi N, Ebrahimpour A, Baradaran A, Rahim RA, Mahyudin NA, Manap MY, Bakar FA and Saari N: Optimization of gamma-aminobutyric acid production by Lactobacillus plantarum Taj-Apis362 from honeybees. Molecules 20: 6654-6669, 2015.

19. Marino A, Bellinghieri V, Nostro A, Miceli N, Tariano MF, Guvenc A and Bisignano G: In vitro effect of branch extracts of Juniperus species from Turkey on staphylococcus aureus biofilm. FEMS Immunol Med Microbiol 59: 470-476, 2010.

20. Barrett E, Ross RP, Fitzgerald GF and Stanton C: Rapid screening method for analyzing the conjugated linoleic acid production capabilities of bacterial cultures. Appl Environ Microbiol 73 : 2333-2337, 2007.

21. Crittenden RG, Martinez NR and Playne MJ: Synthesis and utilisation of folate by yoghurt starter cultures and probiotic bacteria. Int J Food Microbiol 80: 217-222, 2003.

22. Rosberg-Cody E, Stanton C, O'Mahony L, Wall R, Shanahan F, Quigley EM, Fitzgerald GF and Ross RP: Recombinant lactobacilli expressing linoleic acid isomerase can modulate the fatty acid composition of host adipose tissue in mice. Microbiology 157: 609-615, 2011
23. Sreekumar $\mathrm{O}$ and Hosono A: The antimutagenic properties of a polysaccharide produced by Bifidobacterium longum and its cultured milk against some heterocyclic amines. Can J Microbiol 44: 1029-1036, 1998.

24. Barrett E, Ross RP, O'Toole PW, Fitzgerald GF and Stanton C: $\gamma$-Aminobutyric acid production by culturable bacteria from the human intestine. J Appl Microbiol 113: 411-417, 2012.

25. Cryan JF and O'Mahony SM: The microbiome-gut-brain axis: From bowel to behavior. Neurogastroenterol Motil 23: 187-192, 2011.

26. McKay DM and Bienenstock J: The interaction between mast cells and nerves in the gastrointestinal tract. Immunol Today 15 533-538, 1994.

27. Zhao A, Hu X, Pan L and Wang X: Isolation and characterization of a gamma-aminobutyric acid producing strain Lactobacillus buchneri WPZ001 that could efficiently utilize xylose and corncob hydrolysate. Appl Microbiol Biotechnol 99: 3191-3200, 2015

28. Brasca M, Hogenboom JA, Morandi S, Rosi V, D'Incecco P, Silvetti $\mathrm{T}$ and Pellegrino L: Proteolytic activity and production of $\gamma$-aminobutyric acid by streptococcus thermophilus cultivated in microfiltered pasteurized milk. J Agric Food Chem 64: 8604-8614, 2016

29. Tong JC, Mackay IR, Chin J, Law RH, Fayad K and Rowley MJ: Enzymatic characterization of a recombinant isoform hybrid of glutamic acid decarboxylase (rGAD67/65) expressed in yeast. J Biotechnol 97: 183-190, 2002.

30. Binh TT, Ju WT, Jung WJ and Park RD: Optimization of gamma-amino butyric acid production in a newly isolated Lactobacillus brevis. Biotechnol Lett 36: 93-98, 2014 